\title{
Femtosecond pump/supercontinuum-probe spectroscopy: Optimized setup and signal analysis for single-shot spectral referencing
}

A. L. Dobryakov, S. A. Kovalenko, A. Weigel, J. L. Pérez-Lustres, J. Lange, A. Müller, and N. P. Ernsting

Citation: Review of Scientific Instruments 81, 113106 (2010); doi: 10.1063/1.3492897

View online: https://doi.org/10.1063/1.3492897

View Table of Contents: http://aip.scitation.org/toc/rsi/81/11

Published by the American Institute of Physics

\section{Articles you may be interested in}

Accurate convergence of transient-absorption spectra using pulsed lasers

Review of Scientific Instruments 86, 053106 (2015); 10.1063/1.4921479

Femtosecond pump/supercontinuum-probe setup with $20 \mathrm{kHz}$ repetition rate

Review of Scientific Instruments 83, 093105 (2012); 10.1063/1.4750978

High-time-resolution pump-probe system with broadband detection for the study of time-domain vibrational dynamics

Review of Scientific Instruments 78, 103108 (2007); 10.1063/1.2800778

Invited Review Article: Pump-probe microscopy

Review of Scientific Instruments 87, 031101 (2016); 10.1063/1.4943211

Broadband ultraviolet-visible transient absorption spectroscopy in the nanosecond to microsecond time domain with sub-nanosecond time resolution

Review of Scientific Instruments 84, 073107 (2013); 10.1063/1.4812705

Ultrafast optical parametric amplifiers

Review of Scientific Instruments 74, 1 (2003); 10.1063/1.1523642

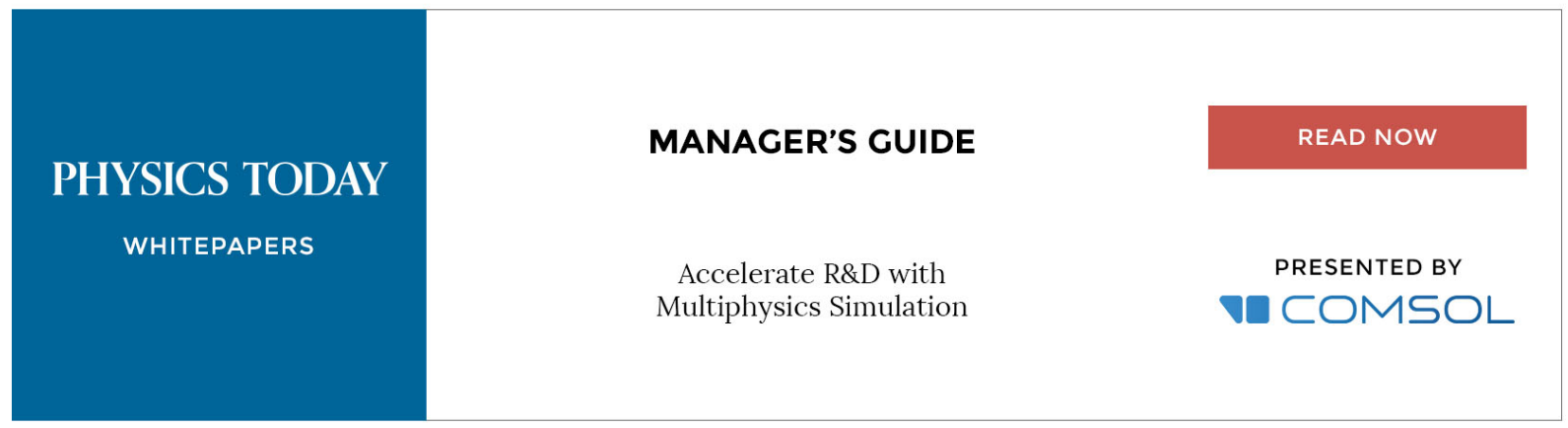




\title{
Femtosecond pump/supercontinuum-probe spectroscopy: Optimized setup and signal analysis for single-shot spectral referencing
}

\author{
A. L. Dobryakov, ${ }^{1}$ S. A. Kovalenko, ${ }^{1}$ A. Weigel, ${ }^{1}$ J. L. Pérez-Lustres, ${ }^{1, a)}$ J. Lange, ${ }^{2}$ \\ A. Müller, ${ }^{2}$ and N. P. Ernsting ${ }^{1}$ \\ ${ }^{1}$ Department of Chemistry, Humboldt University, Brook-Taylor-Str. 2, D-12489 Berlin, Germany \\ ${ }^{2}$ Department of Physics, Martin Luther University Halle-Wittenberg, von-Danckelmann-Platz 3, \\ D-06120 Halle, Germany
}

(Received 29 May 2010; accepted 29 August 2010; published online 16 November 2010)

\begin{abstract}
A setup for pump/supercontinuum-probe spectroscopy is described which (i) is optimized to cancel fluctuations of the probe light by single-shot referencing, and (ii) extends the probe range into the near-uv (1000-270 nm). Reflective optics allow $50 \mu \mathrm{m}$ spot size in the sample and upon entry into two separate spectrographs. The correlation $\gamma_{\text {same }}$ between sample and reference readings of probe light level at every pixel exceeds 0.99 , compared to $\gamma_{\text {consec }}<0.92$ reported for consecutive referencing. Statistical analysis provides the confidence interval of the induced optical density, $\Delta$ OD. For demonstration we first examine a dye (Hoechst 33258) bound in the minor groove of double-stranded DNA. A weak $1.1 \mathrm{ps}$ spectral oscillation in the fluorescence region, assigned to DNA breathing, is shown to be significant. A second example concerns the weak vibrational structure around $t=0$ which reflects stimulated Raman processes. With $1 \%$ fluctuations of probe power, baseline noise for a transient absorption spectrum becomes $25 \mu \mathrm{OD}$ rms in $1 \mathrm{~s}$ at $1 \mathrm{kHz}$, allowing to record resonance Raman spectra of flavine adenine dinucleotide in the $S_{0}$ and $S_{1}$ state. (C) 2010 American Institute of Physics. [doi:10.1063/1.3492897]
\end{abstract}

\section{INTRODUCTION}

Transient optical absorption spectroscopy of molecules has become a common tool of photochemistry and -physics. ${ }^{1-8}$ The use of supercontinuum or "white-light" probe pulses ${ }^{9}$ was instrumental since a wide spectral range can be observed in this way and resolved in time to $100 \mathrm{fs}$ or better. However not only the decay and rise of prominent electronic bands is monitored. More frequently the discussion centers on subtle changes of band shape and mean transition frequency which may indicate excitonic energy transfer, conformational change, or solvation. Corresponding assignments require photometric accuracy and precision which is sufficient to rule out competing interpretations. With this objective in mind we have developed a pump/ supercontinuum-probe spectrometer for use with liquid samples, which is reported here.

We focus on the advantage which single-shot spectral referencing can provide. In this mode, like in a dual-beam spectrometer, each supercontinuum light pulse is split into two identical copies: a sample copy which passes through the solution to be examined, and a reference copy. By comparing their energy point-for-point at every wavelength, a singleshot absorption spectrum can be constructed. Even though such dual beam measurements are not uncommon in transient-absorption spectroscopy, it appears that the best practice and theoretical advantage has not been investigated systematically. This is the purpose of the present paper.

Setups which were described recently ${ }^{10,11}$ use subse-

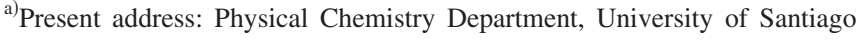
de Compostela E-15782, Santiago de Compostela, Spain.
}

quent sample and reference pulses of white light. They exploit the correlation between successive pulses ${ }^{12,13}$ of whitelight energy in a given wavelength segment. In Ref. 13 the signal/noise $(\mathrm{S} / \mathrm{N})$ ratio was analyzed in terms of this correlation. With our single-shot referencing, a similar formula applies, but the correlation depends on geometrical factors only and no longer directly on laser noise. Thus high precision can be obtained even if successive probe pulses are uncorrelated, for example at long delays (20-1000 ns) in a photobiological cycle. ${ }^{14}$

The paper is organized as follows. In the next section the experimental setup is explained in detail. The third section is devoted to the acquisition of primary data, their transformation into an induced optical density $\Delta \mathrm{OD}(\lambda, t)$, and to the corresponding confidence interval $\mathrm{CI}_{\Delta \mathrm{OD}}$. We conclude with two demonstrations.

\section{SETUP FOR TRANSIENT ABSORPTION}

Two identical setups are used. One is driven by a titanium:saphire laser system which delivers $30 \mathrm{fs}$ fundamental pulses around $800 \mathrm{~nm}$ (Femtolasers sPro). Pulses for continuum generation $(20 \mu \mathrm{J})$ are usually taken from the frequency-doubled beam. Pump pulses $(0.6 \mu \mathrm{J})$ are conveniently generated at 400 or $267 \mathrm{~nm}$, and other pump wavelengths are reached by parametric optical amplification (Light Conversion TOPAS). The other setup is driven by 150 fs fundamental pulses at $775 \mathrm{~nm}$ (Clark-MXR CPA 2001). In this case, $30 \mathrm{fs}$ probe pulses are generated at $520 \mathrm{~nm}$ in a two-stage noncollinear optical parametric amplifier (Jobin Yvon NOPA). The $0.6 \mu \mathrm{J}$ pump pulses are generated in a one-stage NOPA, followed by frequency dou- 


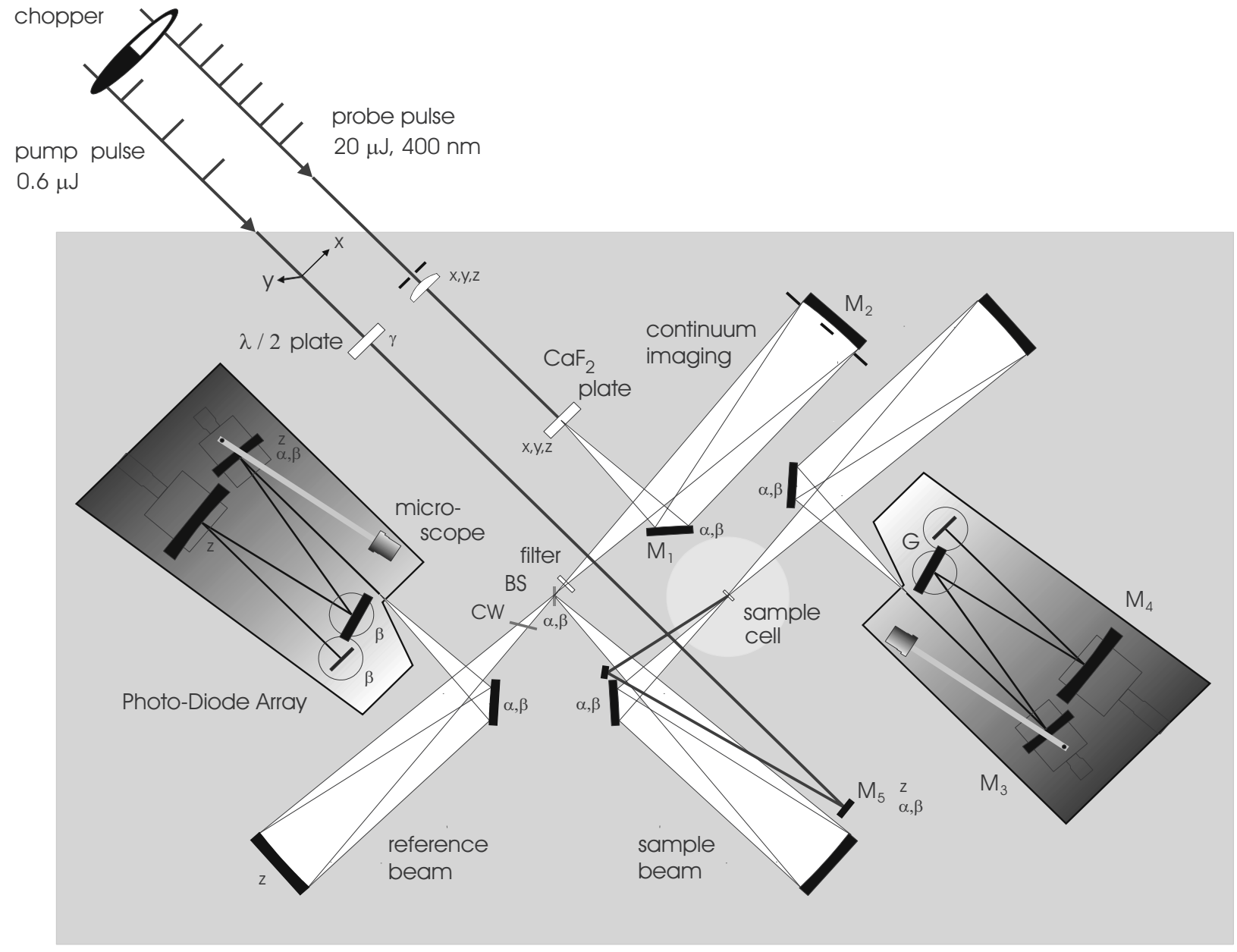

$740 \mathrm{~mm}$

\section{$1200 \mathrm{~mm}$}

FIG. 1. Setup for transient absorption. Multifilament supercontinuum generation extends the probe range from the visible into the uv to $\lambda \approx 270 \mathrm{~nm}$, but it requires optics for a $6^{\circ}$ full solid angle at the source. Mirror objectives allow $50 \mu \mathrm{m}$ spot size in the sample cell and upon entrance into two spectrographs. Apertures and slits are avoided after the BS (for notations see text).

bling when necessary. The laser systems operate with a repetition rate of $1 \mathrm{kHz}$ and every second pump pulse is chopped off. The time delay between pump and probe pulses is controlled by a delay stage (Physik Instrumente M-531.5IM). By placing the stage with its triple mirror into the probe beam, the pump beam can be equipped entirely with dielectric mirrors. Change of pump wavelength is facilitated by a set of different mirrors on each mount.

The transient-absorption spectrograph is sketched schematically in Fig. 1. Supercontinuum light is generated by focusing the probe pulses just before a $1 \mathrm{~mm} \mathrm{CaF}_{2}$ plate with a lens (fused silica $\equiv f . s ., f=200 \mathrm{~mm}$ ). The plate remains stationary during an acquisition sequence (see below), but it is translated in both directions orthogonal to the beam by about $100 \mu \mathrm{m}$ whenever the delay stage moves to a new position. (Rotation of the plate is avoided because of an intensity dependence on rotation angle due to nonlinear interactions. ${ }^{15}$ ) The continuum extends a full angle of about $6^{\circ}$, as found by an aperture stop before field mirror $\mathrm{M}_{2}$. Residual laser light close to the optical axis is absorbed by a central stop. Multifilament generation results in a speckle pattern of about 2-6 homogeneous zones at $\mathrm{M}_{1}$ which should be stable during the acquisition sequence. By optimizing the input beam diameter $(\leq 6 \mathrm{~mm})$ and lens-plate distance, measurement of transient optical density is possible from the near-IR to $270 \mathrm{~nm}$.

Optical relay of the supercontinuum is achieved by repeated use of a 1:1 objective which is specified in Fig. 2. Spherical mirror $\mathrm{M}_{1}$ is convex with radius $R_{1}=+9400 \mathrm{~mm}$ and $\mathrm{M}_{2}$ concave with $R_{2}=-406.4 \mathrm{~mm}$ (Edmund Scientific) in an off-axis Schwarzschild arrangement. ${ }^{16}$ All mirrors are aluminum-coated with enhancement optimized around $300 \mathrm{~nm}$. After replacing the $\mathrm{CaF}_{2}$ plate with a single-mode optical fiber source, the point spread through the system is monitored with a microscope. The first objective images the supercontinuum source $\sim 1 \mathrm{~mm}$ in front of beam splitter BS. About $20 \mathrm{~mm}$ before the BS, the light passes through a color filter which is made with a flow cell $(0.3 \mathrm{~mm}$ internal path length between $0.2 \mathrm{~mm}$ thick $f$.s. windows). BS has approximately $40 \%$ transmission and $40 \%$ reflection for the front surface of a $f$.s. substrate, which has a wedge of $4^{\circ}$. Light reflected from the front surface is used for optical probing and transmitted light for reference, as shown in Fig. 1. In this arrangement, a lateral displacement of the source leads to equivalent displacements on the entrance planes of the two 


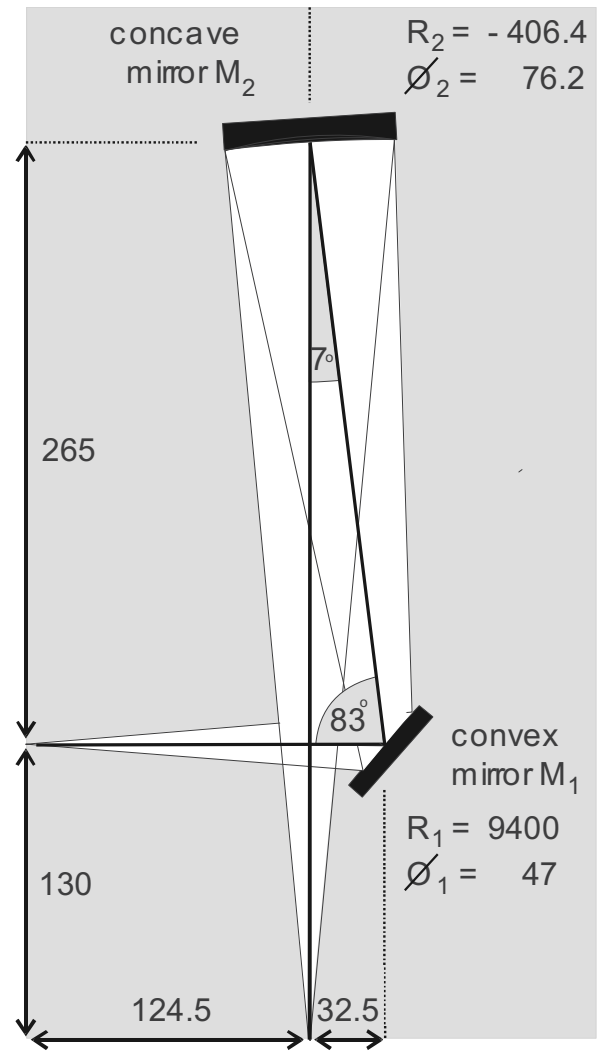

FIG. 2. Specifications of the Schwarzschild objective, as optimized by ray tracing.

spectrographs. A counter-wedge in the reference beam cancels the angular dispersion which is caused by BS, and its partial reflection balances the additional losses in the sample beam. The degrees-of-freedom needed for alignment are also indicated in the figure. $x, y$, and $z$ refers to translation and $\alpha \beta \gamma$ for rotation around axes $x, y$, and $z$, where $z$ corresponds to the direction of beam propagation or to the mirror normal.

The spectrographs employ a Czerny-Turner mount of a ruled plane grating. Compared to imaging gratings, photon loss to unused orders is reduced and the uv efficiency can be maximized by the choice of blaze angle. Field mirrors $\mathrm{M}_{3}$ $(\varnothing 60 \mathrm{~mm})$ and $\mathrm{M}_{4}$ (60 $\mathrm{mm}$ high and $90 \mathrm{~mm}$ wide) have the same radius of curvature $R_{3,4}=-411 \mathrm{~mm}$. For transient absorption we use gratings with about 200 lines/mm (for example Newport 53-346R) to map a wide range of the supercontinuum onto the $1 / 2$ in. photodiode-array (see below). Each spectrograph is equipped with a microscope which can be swiveled into the beam. It allows to view the supercontinuum, which for this purpose must be attenuated, and to center it on the input position. The latter is defined by a pinhole of $50 \mu \mathrm{m}$ diameter which can be moved reproducibly in and out of the entrance plane. In this way one can also ascertain that a source point spreads to less than $50 \mu \mathrm{m}$ diameter. During measurement the pinholes are removed, and the effective entrance slits are given by the position of the source on the $\mathrm{CaF}_{2}$ plate. The procedure epitomizes our concept: to avoid all apertures and slits after the BS, since they act as spectral filters which are not controlled and hence, will not be equivalent. With the sample microscope

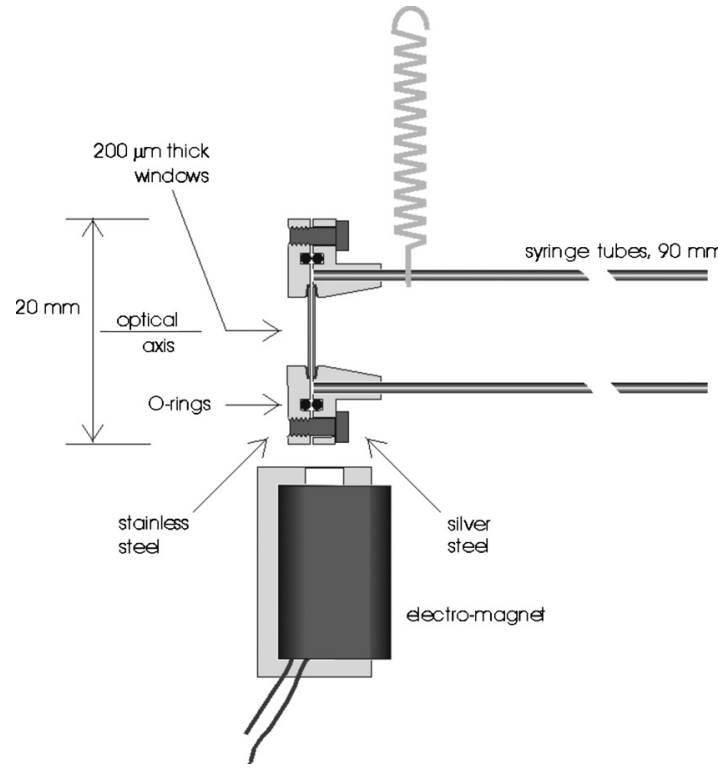

FIG. 3. Sample cell of variable optical path length $\leq 0.5 \mathrm{~mm}$. While the solution is flown through slowly, the cell is oscillated at its resonance frequencies (ca. $20 \mathrm{~Hz}$ ) in the two directions orthogonal to the optical axis.

the pump-probe overlap region in the flow cell is also monitored. The $45^{\circ}$ arrangement of the beam lines has two advantages: one can look equally well into both microscopes, and the sample cell (see below) is accessed easily.

The choice of two spectrographs with their separate detector arrays deserves comment, as does routing of the reference beam past the sample cell. We have tried many alternative combinations over a decade (see for example Refs. 17 and 23) and on balance found only minor gains of $\mathrm{S} / \mathrm{N}$, at the cost of crowding at the sample cell, and lack of flexibility upon direct coupling into the spectrograph(s). In our experience, the geometry of Fig. 1 provides best accessibility of the sample cell (cryostats, multiple pump pulses, etc.) and easiest wavelength mapping between the detector arrays. ${ }^{18}$

The sample cell is a critical part of the setup. Fig. 3 shows a cell of small volume $(15 \mu \mathrm{l})$ which allows to exchange liquid sample between shots completely. It consists of two turned flanges, each with a window hole and a groove for an O-ring. Windows of fused silica, $200 \mu \mathrm{m}$ thick and polished to highest grade, are glued into each flange with silicon glue. A Teflon spacer between the two components is cut so that sample liquid can flow from the inlet to the outlet port. These ports are provided by long syringe tubes which are pressed into one flange. The tubes also support the cell such that it can oscillate orthogonally to the optical axis. Two electromagnets drive the oscillations to an amplitude of $\approx 2 \mathrm{~mm}$ at the resonance frequencies (typically 20 and $22 \mathrm{~Hz}$ ). In this way successive shots are placed $\approx 250 \mu \mathrm{m}$ apart on fresh sample and window. On a longer time scale even viscous solutions, such as dyes in ionic liquids or proteins concentrated in water, can be exchanged by slow flow. ${ }^{19}$

Inside the spectrographs we use NMOS linear image sensors with 512 pixels and a pixel pitch of $25 \mu \mathrm{m}$ (Hamamatsu S3904-512Q). The pixel height of $2.5 \mathrm{~mm}$ is necessary to capture the entire spectrum, which becomes 


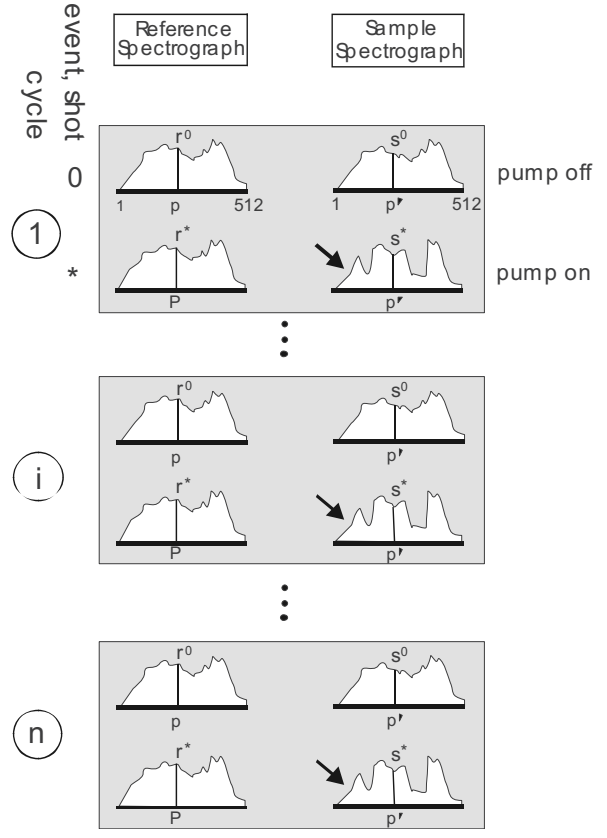

FIG. 4. Acquisition sequence and corresponding data package for a fixed delay position. $p 1=p 2$ pixel considered in the reference and sample spectrograph, $r, s$ corresponding readout. During a sequence the $\mathrm{CaF}_{2}$ plate is not moved.

$1.5 \mathrm{~mm}$ high at the edges due to astigmatism. The precision driver circuit $\mathrm{C} 7884$ gives a boxcar waveform output with low noise and good output linearity. Although specified for data rates up to $500 \mathrm{kHz}$ only, the driver can be tuned to the $512 \mathrm{kHz}$ master clock which is required. An image sensor controller has to create this master clock for reading out the sensor lines, to synchronize it with the laser trigger and the chopper state, and also to generate the clock signal for analog-digital conversion. For this we use an acquisition card with four simultaneous 14-bit AD channels at $2.5 \mathrm{MS} / \mathrm{s}$ (National Instruments NI-PCI-6132). We find it imperative that the analog signals can also be monitored on a scope, to minimize baseline noise by fine adjustments between measurements. The controller is based on a complex programmable logic device, allowing to change the sensors (e.g., to S39041024Q).

\section{ON FORMING THE RATIO OF SAMPLE AND REFERENCE DATA}

The acquisition sequence for a fixed delay position is explained with the help of Fig. 4. The example for this section was recorded with the Femtolasers system, in which case white-light generation is relatively noisy $(\approx 10 \%$ intensity fluctuations from shot to shot) so that the merit of singleshot referencing becomes apparent directly. Pixel numbers are denoted $p, p^{\prime}$ on the reference and sample spectrograph as shown in the figure, and the corresponding photodiode values are $r$ and $s$. As the spectra are registered, we distinguish two events: either the pump beam is off (event or shot 0 ) or else it is on $(*)$. It does not matter for the present discussion whether the pump is physically present on the sample during event $*$ or not; for example when a $\Delta \mathrm{OD}$ baseline is being recorded the pump beam must be blocked.

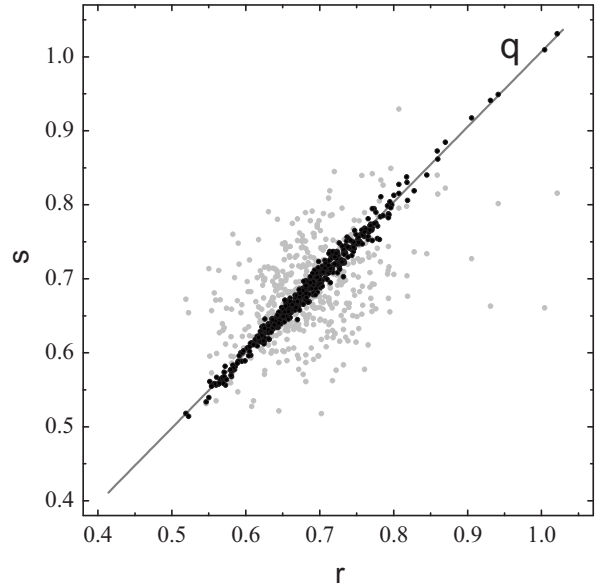

FIG. 5. Correlation between reference $r$ and sample reads $s$, at the pixel considered, for 500 events like in Fig. 6. Black points: same continuum shot, registered in parallel by the two spectrographs. The regression line through the origin, with slope or optimal quotient $q$, is also shown. Gray points: successive continuum shots, registered by the sample spectrograph only.

In the figure the presence of the pump beam is symbolized by an arrow impinging on the sample spectrum. Sometimes the counts on the photodiodes are indexed by the event, for example $\left\{r^{0}, s^{0}\right\}$ or $\left\{s^{0}, s^{*}\right\}$. Successive events 0 and $*$ are grouped into an individual measurement cycle with running index $i$; a package consists of $n$ cycles.

The construction of a transient absorption spectrum $\Delta \mathrm{OD}(\lambda, t)$ is discussed next. Imagine that a data package has been recorded for the delay time $t$. We can then set $p$ and $p^{\prime}$ (which may be different) and look at the corresponding $r$ and $s$. This can be done for the $n$ pairs of spectra which represent event 0 , and equally for the $n$ pairs of spectra which represent event $*$. Let us consider only one such case; the various $\left\{r_{i}, s_{i}\right\}$ are shown in Fig. 5 as black points. We seek the line which goes through the origin and minimizes the squared distances to the data points (note that this prescription differs from the regression when there is no uncertainty in the $r$ values). The optimal slope or quotient $q$ (to be distinguished from individual quotients $q_{i}=s_{i} / r_{i}$ ) is given by

$$
q=\frac{\sum s_{i}^{2}+\sqrt{4 \sum r_{i} s_{i}+\left(\sum r_{i}^{2}-\sum s_{i}^{2}\right)^{2}}-\sum r_{i}^{2}}{2 \sum r_{i} s_{i}} .
$$

This procedure is performed for pump off and pump on, so that $q^{0}$ and $q^{*}$ are obtained.

The statistical data $r_{i}$ and $s_{i}$ have averages $\bar{r}$ and $\bar{s}$ and variances

$$
\sigma_{r}=\sqrt{\sum\left(r_{i}-\vec{r}\right)^{2} /(n-1)}
$$

and

$$
\sigma_{s}=\sqrt{\sum\left(s_{i}-\bar{s}\right)^{2} /(n-1)} .
$$

It will be useful to look at the $r, s$-correlation

$$
\gamma=\frac{\sum r_{i} s_{i}-n \overline{r s}}{(n-1) \sigma_{r} \sigma_{s}} .
$$

Altogether $n=500$ cycles were recorded for the example package, requiring $1 \mathrm{~s}$ acquisition time. The black points $\left\{r_{i}^{0}, s_{i}^{0}\right\}$ in Fig. 5 refer the same event 0, i.e., the shots are 
used to define $100 \%$ transmission with the pump off. The optimal quotient $q$ is also shown (black line); the correlation $\gamma$ is 0.992 in this case. (Note that $p=p^{\prime}=275$ here. The correlation drops by $0.005 \Delta p^{2}$ when pixels are detuned and becomes zero for $|\Delta p| \geq 30$, or $\Delta \widetilde{\nu} \geq 1200 \mathrm{~cm}^{-1}$. Sample and reference wavelengths were mapped onto identical pixel numbers by making use of this correlation.) With a single spectrograph one would record $\left\{s_{i}^{0}, s_{i}^{*}\right\}$ which are shown as gray points, for which the correlation drops to 0.46 . In either case we find normalized noise $\sigma_{r} / \bar{r} \approx \sigma_{s} / \bar{s}=9.6 \%$. These values will be used to discuss the merit of single-shot referencing.

Let us consider the distribution when the two spectra (and therefore the photodiode readouts at $p=p^{\prime}=275$ ) are taken from the same shot, i.e., by parallel readout of reference and sample spectrographs (black points in Fig. 5). It is governed by a joint probability distribution

$$
P(r, s)=\frac{1}{2 \pi \sqrt{\operatorname{det} \mathbf{C}}} \exp \left(-\frac{1}{2} \mathbf{d}^{T} \mathbf{C}^{-1} \mathbf{d}\right) .
$$

Here $\mathbf{C}$ is the correlation matrix

$$
\mathbf{C}=\left[\begin{array}{cc}
\sigma_{r}^{2} & \gamma \sigma_{r} \sigma_{s} \\
\gamma \sigma_{r} \sigma_{s} & \sigma_{s}^{2}
\end{array}\right],
$$

and

$$
\mathbf{d}=\left[\begin{array}{l}
r-\bar{r} \\
s-\bar{s}
\end{array}\right] .
$$

Now consider for a moment only one variable, for example $r$. The question is: how well do the experimental averages $\bar{r}$ approach the true average $\mu_{r}$ ? How large is the confidence interval $\mathrm{CI}_{r}$ such that $\bar{r}$, from a data package with $n$ cycles, falls into the range $\mu_{r} \pm \mathrm{CI}_{r}$ for a given confidence? This is equivalent to the confidence of finding $\mu_{r}$ in the experimental range $\bar{r} \mp \mathrm{CI}_{r}$. The experimental distribution of the $r_{i}$ is stepped in a coarse manner for small $n$. But as $n$ becomes larger, the stepping in the experimental curve gradually disappears and the histogram approaches the smooth true or parent distribution. Because the distribution is defined ever more precisely, the determination of $\mu_{r}$ becomes more and more accurate if no systematic errors are present. This is the essence of data averaging ${ }^{20}$

$$
\mathrm{CI}_{r}=\sigma_{r} / \sqrt{n} \text {. }
$$

Standard error of the ratio $s / r$. Returning to the many pairs $\left\{r_{i}, s_{i}\right\}$, we form the corresponding individual quotients $q_{i}$ $\equiv s_{i} / r_{i}$. If the parent distribution is approximated by $P(r, s)$, then what are the expectation value and standard deviation of the quotient, and how large is $\mathrm{CI}_{q}$ for $68 \%$ confidence when $\mathrm{n}$ cycles are measured? The deviation of the quotient

$$
\delta_{q}=s / r-\bar{s} / \bar{r}
$$

is plotted in Fig. 6 as a contour map of gray lines. The quotient-and therefore also the square deviation $\delta_{q}^{2}$-is sampled with $P(r, s)$ [Eqs. (4)-(6)] which is also shown in the figure. We have to form the integral

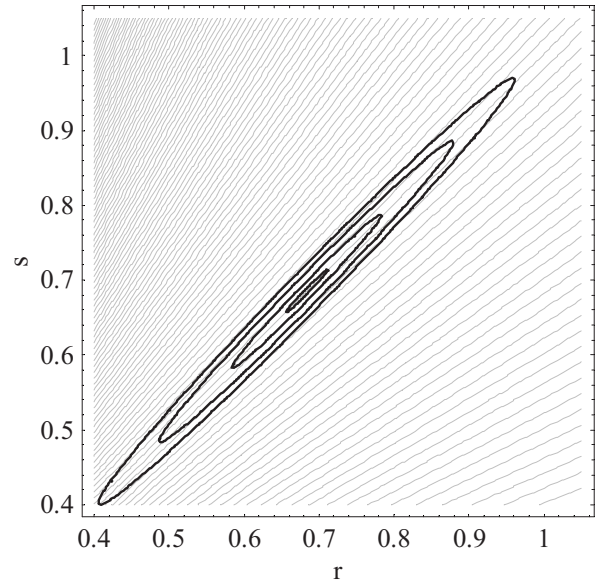

FIG. 6. Parent distribution for the data in Fig. 5 (black contours at $10^{-4}$, $10^{-2}, 0.3,0.9$ of the peak) sampling the deviation $\delta_{q}$ of the slope. (Along gray lines the corresponding $\delta_{q} \equiv$ constant. $\delta_{q} \equiv 0$ corresponds approximately to the long axis).

$$
\sigma_{q}^{2}=\iint \delta_{q}^{2}(r, s) P(r, s) d r d s .
$$

If the correlation were 1 , the parent distribution would sharply sit on the line for which $\delta_{q}^{2}(r, s)=0$. In this limiting case, even if the shots fluctuate wildly the quotient keeps constant. By Taylor expansion around $\bar{r}, \bar{s}$ to second order we approximate

$$
\delta_{q}^{2} \approx \frac{1}{2} D_{r r}(r-\bar{r})^{2}+D_{r s}(r-1)(s-1)+\frac{1}{2} D_{s s}(s-\bar{s})^{2},
$$

where

$$
\begin{aligned}
& D_{r r}=2 \bar{s}^{2} / \bar{r}^{4}, \\
& D_{s s}=2 / \bar{r}^{2}, \\
& D_{r s}=-2 \bar{s} / \bar{r}^{3} .
\end{aligned}
$$

The approximation is reasonable for relative variances up to $10 \%$. Integration as in Eq. (9) gives

$$
\sigma_{q}^{2} \approx \frac{\bar{s}^{2}}{\bar{r}^{4}} \sigma_{r}^{2}-\frac{2 \gamma \bar{s}}{\bar{r}^{3}} \sigma_{r} \sigma_{s}+\frac{1}{\bar{r}^{2}} \sigma_{s}^{2} .
$$

Like in Eq. (7) the confidence interval for the ratio $s / r$ becomes

$$
\mathrm{CI}_{q}=\sigma_{q} / \sqrt{n} .
$$

If the two light beams can be balanced, $\bar{s} \approx \bar{r}$ and $\sigma_{s} \approx \sigma_{r}$ so that

$$
\mathrm{CI}_{q} \approx \frac{\sigma_{r}}{\bar{r}} \sqrt{2(1-\gamma) / n} .
$$

Confidence interval of a transient absorption spectrum. We refer to Fig. 5 and assume that the measurement gives optimal quotients $q^{0}$ and $q^{*}$ for the pump-off and pump-on plots of $s$ versus $r$, respectively. The induced optical density at the chosen wavelength is

$$
\Delta \mathrm{OD}=-\log \left(q^{*} / q^{0}\right) .
$$

Note that this definition of $\Delta \mathrm{OD}$ involves weighted averages of quotients $s_{i} / r_{i}$, with weights proportional to the reference 


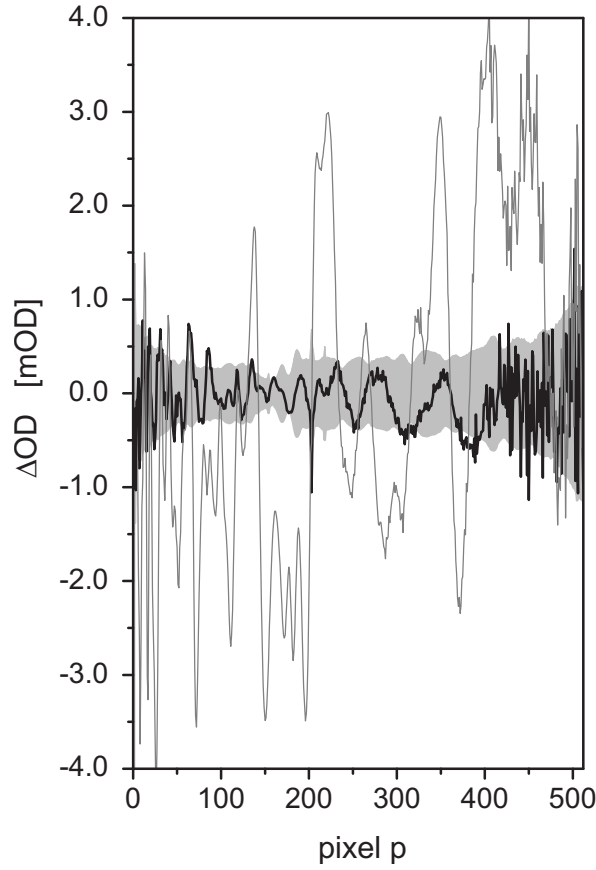

FIG. 7. Change of optical density between events 0 ("pump off") and * ("pump on") but with the pump beam physically blocked. This $\Delta \mathrm{OD}$ baseline (black) has rms noise of $0.33 \times 10^{-3}$ across the full pixel range (wavelengths $277-660 \mathrm{~nm}$ ). The same standard error for $\Delta \mathrm{OD}$ is obtained from regression analysis at each pixel individually (gray band between $\left.\pm \mathrm{CI}_{\Delta \mathrm{OD}}\right)$. The baseline from consecutive shots is shown for comparison (gray line); the improvement of $\mathrm{S} / \mathrm{N}$ by single-shot referencing is $>8$ for this experiment.

light level $r_{i}$ (different from the usual definition which employs an unweighted average, $\left\langle s_{i} / r_{i}\right\rangle,{ }^{13}$ also $\left\langle s_{i}\right\rangle /\left\langle r_{i}\right\rangle$ is used in practice.) Confidence intervals $\mathrm{CI}_{q}^{0}$ and $\mathrm{CI}_{q}^{*}$ [Eqs. (13) and (14)] are associated with the determination of the two quotients. If the determinations are not correlated we obtain, as main result, the confidence interval for $\Delta \mathrm{OD}$

$$
\mathrm{CI}_{\Delta \mathrm{OD}}=\frac{1}{\ln (10)} \sqrt{\left(\frac{\mathrm{CI}_{q}^{0}}{q^{0}}\right)^{2}+\left(\frac{\mathrm{CI}_{q}^{*}}{q^{*}}\right)^{2}}
$$

Here $\log$ and $\ln$ denote the decadic and natural logarithm, respectively. A useful formula is obtained when the beams are balanced, $q=1$. In this case $\mathrm{C}_{q}^{0} \approx \mathrm{C}_{q}^{*}$ so that

$$
\mathrm{CI}_{\Delta \mathrm{OD}} \approx \frac{\sigma_{r}}{\bar{r} \sqrt{n}} \sqrt{(1-\gamma)} \frac{\sqrt{2}}{\ln (10)} .
$$

(This expression was used ${ }^{11,13}$ for referencing by every second shot with a single spectrograph, in which case $\gamma$ refers to the correlation between the various $s_{i}^{*}$ and $s_{i}^{0}$, and in Eq. (8) one has $\delta=s^{*}-s^{0}$ ).

These results are now applied to the set of spectra recorded with the Femtolasers system with the pump beam physically blocked. The resulting baseline is shown solid black in Fig. 7; we find zero mean and rms deviation of $0.33 \times 10^{-3}$, consistent with the estimate $0.24 \times 10^{-3}$ [Eq. (16)] at the single pixel $p=275$. The confidence interval [Eq. (16)] is shown as a gray band in the figure. Successive records $\left\{s_{i}^{0}, s_{i}^{*}\right\}$ from the sample channel alone were shown in Fig. 5 as gray points; their correlation is $\gamma_{\text {consec }} \approx 0.5$. When these are used as in competing setups, ${ }^{10,11}$ then the gray base- line is produced. By comparison, with $\gamma_{\text {same }}=0.992$ we find an eightfold improvement of $\mathrm{S} / \mathrm{N}$ by same-shot referencing for our example. However comparisons between different laboratories can also be made on the basis of Eq. (17). The highest correlation between successive shots reported to date is $\gamma_{\text {consec }}=0.916{ }^{10,21}$ Abandoning the best consecutive referencing for single-shot referencing can improve $\mathrm{S} / \mathrm{N}$ by a factor of 3 .

\section{APPLICATIONS}

The baseline noise of transient absorption measurements [Eq. (17)] depends also on the "laser noise" $\sigma_{r} / \bar{r}$ of the probe light, to be multiplied with the correlation factor $\sqrt{1-\gamma}$ which was discussed before. When the precision achieved by different setups is compared, the product of both factors tends to be considered; the laser system becomes part of the assessment of the femtosecond spectrograph. This is why we present applications which were measured with different probe noise: (1) using the Femtolasers system as before, and (2) with a NOPA system as in competing setups. ${ }^{10,11}$

\section{A. A weak spectral oscillation of a photoexcited DNA-dye complex ${ }^{22}$}

The bis-benzimidazole dye Hoechst 33258 (H33258) binds in the minor groove of duplex DNA, for example $\mathrm{d}(\text { CGCAAATTTGCG })_{2}$, to A:T-rich regions. Steady-state optical spectra of the complex are shown in Fig. 8(a). Transient absorption spectra $\Delta \operatorname{OD}(\lambda, t)$ are shown in Fig. 8(b) for the period of 180-1980 fs. Negative signal around $350 \mathrm{~nm}$ is due to ground-state bleaching and positive signal around $550 \mathrm{~nm}$ to excited-state absorption (ESA). Stimulated emission (SE) has $\Delta \mathrm{OD}<0$ in the fluorescence region, but it is overcompensated by ESA and therefore not recognized directly. The change $\Delta \Delta \mathrm{OD}$ from the spectrum at $180 \mathrm{fs}$ is shown in Fig. 8(c); it has a broad dispersive shape with an apparent isosbestic point at $447 \mathrm{~nm}$. From independent measurements, using time-gated broadband fluorescence upconversion, we found that (i) the spectral change comes largely from the SE band shifting to the red, and (ii) the timedependent Stokes shift of the band has a weak oscillation with $\nu \approx 1 \mathrm{ps}^{-1}$ corresponding to $\widetilde{\nu} \approx 30 \mathrm{~cm}^{-1}$. By comparison with molecular-dynamics simulations, this motion is assigned to collective opening and closing of the minor groove. $^{22}$ But can the oscillation also be seen in the transient absorption spectra? To answer this question we fit the data globally, i.e., for all $\lambda_{i}$, by exponential time functions with reasonably distributed decay constants. The residuals from the fit are then discussed with the help of the confidence interval $\mathrm{CI}_{\triangle \mathrm{OD}}$.

The data $\Delta \mathrm{OD}\left(\lambda_{i}, t_{k}\right) \equiv M_{i k}$ constitute a matrix $\mathbf{M}$, with $325 \leq \lambda_{i} \leq 600 \mathrm{~nm}$ and $-0.5 \leq t_{k} \leq 9.0 \mathrm{ps}$ (20 fs steps) in the present case. The transient spectra are stored in the columns of $\mathbf{M}$, and the dimensions of this matrix are denoted $n w l$ and $n t m$ (typically after clipping spectral wings $n w l \approx 480$, and $n t m \approx 400$ ). Coherent signal peaks at $t=0$ as pump and probe overlap in time, and sequential signal rises around $t=0$. For fitting we use the basic time functions 

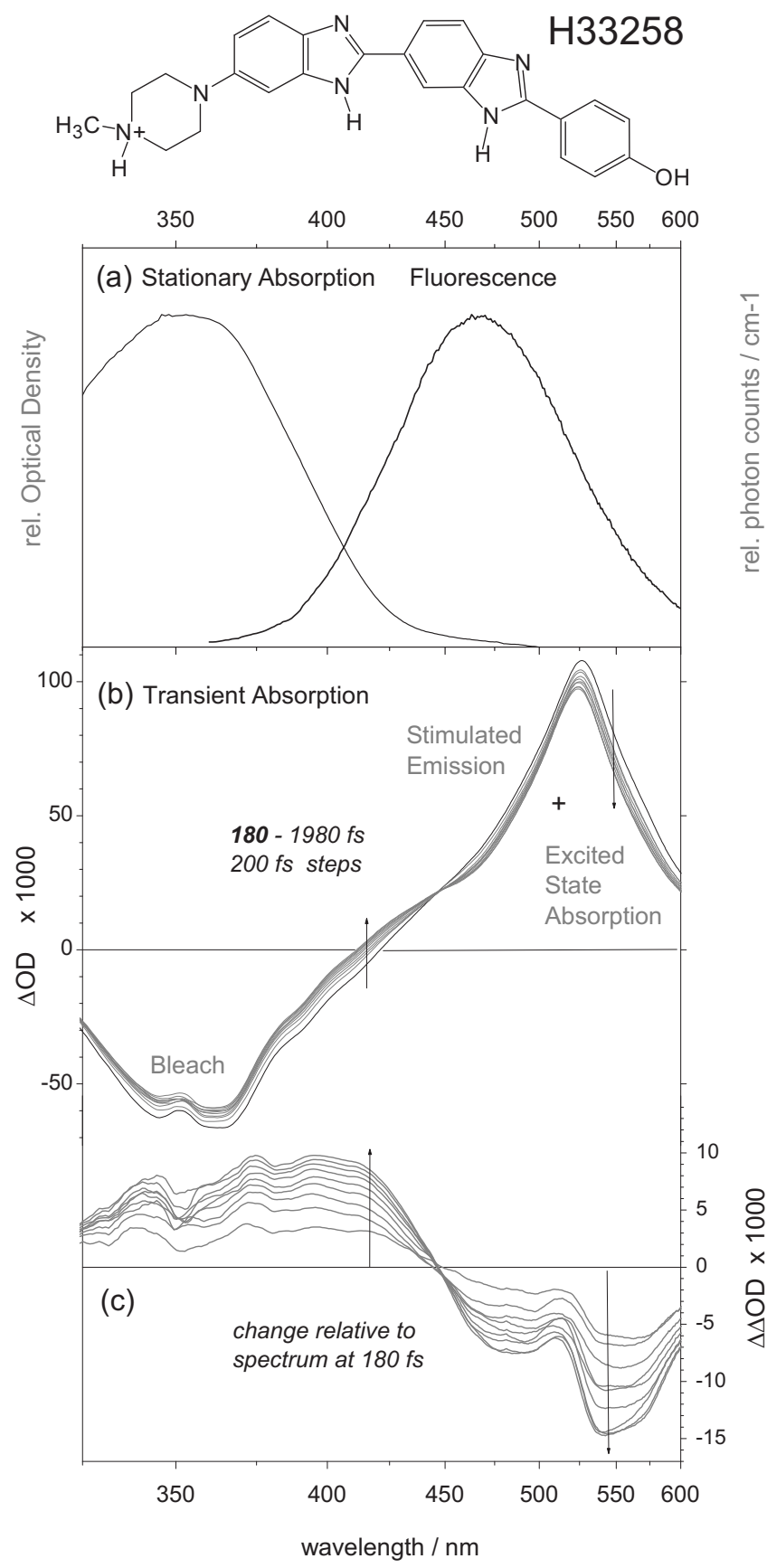

FIG. 8. Optical spectra of the H33258/DNA complex in an aqueous solution, stationary (a) (solid lines) and transient (b) and (c) after femtosecond laser excitation (parallel pump-probe polarization). The evolution in (c) reflects that of stimulated emission which is strictly related to spontaneous fluorescence from the excited ligand $\mathrm{H} 33258$.

$$
\begin{aligned}
G= & 1 / \sqrt{2 \pi d^{2}} \exp \left[-1 / 2(t / d)^{2}\right] \\
& \text { and time derivatives } G^{\prime}, G^{\prime \prime},
\end{aligned}
$$$$
\mathrm{CS}=1 / 2 \operatorname{Erfc}\left(-t / \sqrt{2 d^{2}}\right)
$$$$
\operatorname{CExp}_{m}=1 / 2 \exp \left[1 / 2\left(k_{m} t\right)^{2}-k_{m} t\right] \operatorname{Erfc}\left[\left(d^{2} k_{m}-t\right) / \sqrt{2 d^{2}}\right] .
$$

Here CS and CExp are the Gauss-convoluted step-up function and exponential function $(m=1,2,3)$, respectively. ${ }^{23}$ The pulse-width parameter $d=28$ fs is found by fitting the pure solvent signal ${ }^{24}$ which was recorded in $6 \mathrm{fs}$ steps. The fit function becomes

$$
\begin{aligned}
\Delta \mathrm{OD}\left(\lambda_{i}, t_{k}\right)= & A\left(\lambda_{i}, 1\right) G\left(t_{k}\right)+A\left(\lambda_{i}, 2\right) G^{\prime}\left(t_{k}\right) \\
& +A\left(\lambda_{i}, 3\right) G^{\prime \prime}\left(t_{k}\right)+A\left(\lambda_{i}, 4\right) \mathrm{CS}\left(t_{k}\right) \\
& +A\left(\lambda_{i}, 5\right) \operatorname{CExp}_{1}\left(t_{k}\right)+A\left(\lambda_{i}, 6\right) \operatorname{CExp}_{2}\left(t_{k}\right) \\
& +A\left(\lambda_{i}, 7\right) \operatorname{CExp}_{3}\left(t_{k}\right)
\end{aligned}
$$

The $A\left(\lambda_{i}, j\right)$ are linear coefficients which need not be considered explicitly (see below). Only the nonlinear parameters $k_{1}, k_{2}, k_{3}$ in the time functions must be optimized in a gradient search. For this purpose one guesses their values and forms the matrix $\mathbf{T}_{\text {guess }}$ consisting of the rows $G\left(t_{k}\right), G^{\prime}\left(t_{k}\right), G^{\prime \prime}\left(t_{k}\right), \mathrm{CS}\left(t_{k}\right), \operatorname{CExp}_{1}\left(t_{k}\right), \operatorname{CExp}_{2}\left(t_{k}\right), \operatorname{CExp}_{3}\left(t_{k}\right)$, where $k=1, \ldots n t m$ numbers the columns. The optimal coefficients which are associated with these time functions are then obtained in the $n w l x 7$ matrix $^{25}$

$$
\mathbf{A}=\mathbf{M} \text { pseudoinverse }\left(\mathbf{T}_{\text {guess }}\right) \text {. }
$$

The approximation to the data becomes

$$
\mathbf{M}_{\text {guess }}=\mathbf{A} \mathbf{T}_{\text {guess }} \text {. }
$$

The root-mean-square deviation $\Sigma$ to the data is calculated from the difference $\mathbf{M}-\mathbf{M}_{\text {guess. }}$. In the search, $\Sigma$ is minimized by varying $k_{1}-k_{3}$. The spectra which are associated with the time functions of Eq. (18) are then found in the columns of A.

To locate an oscillation on a 1 ps time scale, the data were treated in two steps. First the time range $1.5 \geq t$ $\geq 9$ ps was fitted with two exponentials only, resulting in optimal $k_{2}=0.79$ and $k_{3}=0.12 \mathrm{ps}^{-1}$. In a second step the entire time range was fitted involving three exponentials but with $k_{2}, k_{3}$ fixed to the previous values, providing an optimal $k_{1}=9.38 \mathrm{ps}^{-1}$. Note that the $k_{1}, k_{2}, k_{3}$ values are correlated and other triples describe $\mathbf{M}$ equally well, but the arguments below are not affected by this correlation. The quality of the global fit can be seen in Fig. 9, where the row at $\lambda$ $=405 \mathrm{~nm}$ of $\mathbf{M}$ (black line) and of optimal $\mathbf{M}_{\text {guess }}$ are compared (lower panel). The fit-curve is shown decomposed into the coherent "pulse" part (red) and the sequential "molecular evolution" part (green), which alone describes the data for $t \geq 180$ fs. Subpicosecond oscillations are directly visible on the experimental curve. Residuals are expanded in the upper panel. Now a weak undulation around the fit, with a broad hump around $1.3 \mathrm{ps}$, emerges. One suspects that this oscillation is an artifact of the global fit with three exponentials only; it might disappear when more exponentials are offered. We therefore augmented the existing set of decay functions by a fourth exponential and searched for the corresponding $k_{4}$, starting systematically from values in the range of $5-0.2 \mathrm{ps}^{-1}$. Also fixed values such as $k_{4}=0.5$ or $2 \mathrm{ps}^{-1}$ were tried. But the hump-and-valley structure of the residuals was not affected, only its distribution around the fit. We conclude that the oscillation is an inherent feature of this particular measurement; ${ }^{26}$ it remains to see whether or not it is significant.

Noise and confidence are therefore discussed at last. Time scans had been carried out with 100 cycles at each 


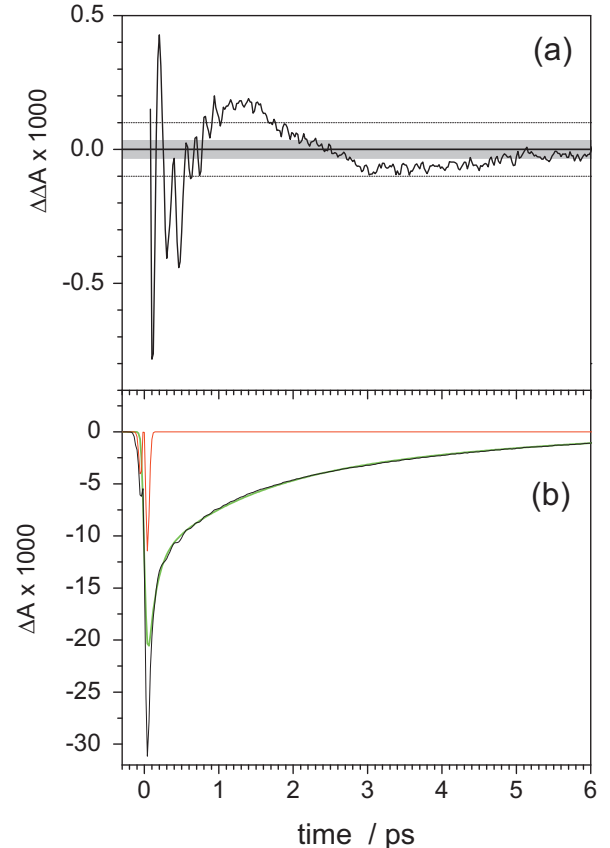

FIG. 9. (Color) Transient absorption trace at $405 \mathrm{~nm}$ (black line in lower panel (b) and its description by a global fit with three convoluted exponentials (green) and Gaussian functions $\mathrm{G}, \mathrm{G}^{\prime}, \mathrm{G}^{\prime \prime}$ (red, see text). The residuals from this description are shown in the upper panel (a). The gray band/ dashed lines represent the range for $68 / 99.7 \%$ confidence.

delay position and the results of 44 scans were averaged; hence $n=4400$. Using $\gamma=0.992$ and $\sigma_{r} / \bar{r}=0.096$ as before, we obtain [Eq. (17)] $\mathrm{CI}_{\Delta \mathrm{OD}}=0.112 \mathrm{mOD}$. Measurements after the pump pulse are also affected by fluctuations of pulse energy. For example at the ESA peak, the deviation from the global fit is $0.39 \mathrm{mOD}$ rms for this reason. On the other hand around $410 \mathrm{~nm}$ the induced optical density is small or zero. Here pump pulse fluctuations are ineffective: fit and measurement have again $0.11 \mathrm{mOD}$ rms deviation at later time $(t>4 \mathrm{ps})$ when the oscillation has subsided. Actually in Fig. 9(a) we show the residuals averaged over 11 traces around $405 \mathrm{~nm}$. The $68 \%$ confidence band between $\pm 0.033 \mathrm{mOD}$ is indicated gray in the figure and the $99.7 \%$ confidence band by dashed lines. The oscillation amplitude exceeds the $99.7 \%$ confidence range and is therefore significant.

\section{B. Femtosecond stimulated Raman scattering of flavine adenine dinucleotide (FAD)}

When multiple excitation pulses cooperate to prepare a transient absorption spectrum, the speed afforded by singleshot spectral referencing becomes an important aspect of the measurement. For example consider femtosecond stimulated Raman scattering ${ }^{27}$ which allows to measure the transient Raman spectrum of an evolving molecular excited state $S_{1}$. Figure 10 shows stimulated Raman spectra of FAD in water. Vibrational bands, typically with amplitudes $<1 \mathrm{mOD}$, originally reside on a broad transient-absorption background which has been subtracted. For their determination the fluctuations $\sigma_{r} / \bar{r}$ of the supercontinuum probe intensity were reduced to the $1 \%$ range by using the Clark-MXR CPA 2001+NOPA laser system. "Actinic" excitation

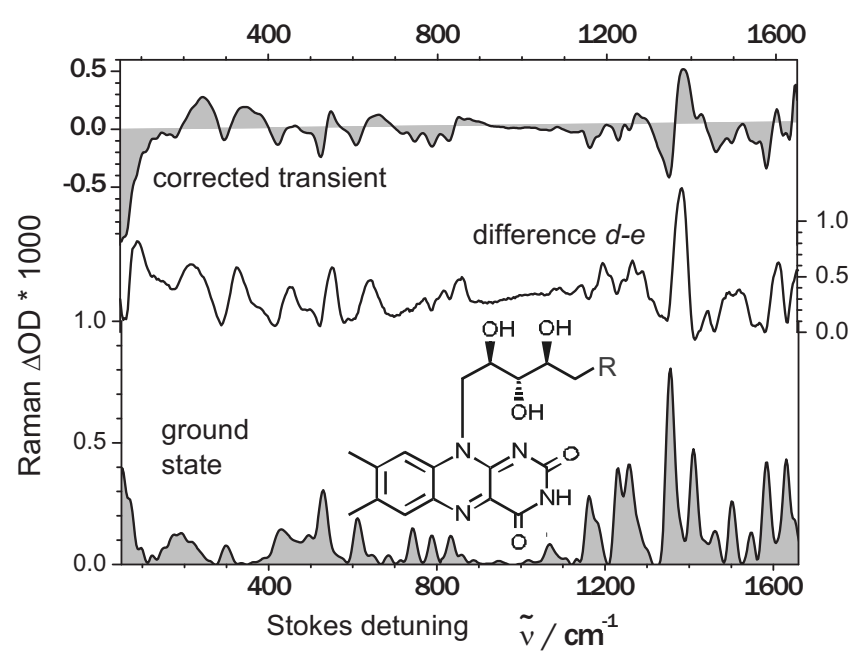

FIG. 10. Femtosecond stimulated Raman scattering spectra of aqueous FAD (inset, with $R=$ adenine diphosphate) constructed from five partial spectra (see text) for the electronic ground state $S_{0}$ (bottom), and for the $S_{1}$ state $0.5 \mathrm{ps}$ after actinic excitation at $475 \mathrm{~nm}$ (top). The Raman pump at $523 \mathrm{~nm}$ was resonant with the $S_{n} \leftarrow S_{1}$ and $S_{1} \rightarrow S_{0}$ transitions, leading to dispersive line shapes. Middle: in the difference spectrum $d$-e most of the population background has been canceled, and the $20 \mu \mathrm{OD}$ noise becomes visible.

$S_{1} \leftarrow S_{0}$ was provided by $30 \mathrm{fs}, 475 \mathrm{~nm}$ pulses. Narrow-band $\left(20 \mathrm{~cm}^{-1}\right)$ "Raman pump" pulses were applied either at $19100 \mathrm{~cm}^{-1}$ ("Raman1") or at $19140 \mathrm{~cm}^{-1}$ ("Raman 2"). They are resonant with the red-edge of the FAD absorption band and can also stimulate the emission; the corresponding population changes contribute to the background signal. An individual measurement cycle consisted of five events as follows:

\begin{tabular}{lcccc} 
Event & $\begin{array}{c}\text { Actinic } \\
\text { pump }\end{array}$ & $\begin{array}{c}\text { Raman } \\
\text { pump 1 }\end{array}$ & $\begin{array}{c}\text { Raman } \\
\text { pump 2 }\end{array}$ & Probe \\
\hline$a$ & 0 & 0 & 0 & 1 \\
$b$ & 0 & 1 & 0 & 1 \\
$c$ & 0 & 0 & 1 & 1 \\
$d$ & 1 & 1 & 0 & 1 \\
$e$ & 1 & 0 & 1 & 1
\end{tabular}

From the corresponding spectra the ground- and excitedstate Raman spectrum can be constructed. ${ }^{28,29}$ With $n$ $=50$ cycles in a data package and averaging 14 scans (total acquisition time $3.5 \mathrm{~s}$ for this cycle) the noise is predicted to be $\approx 20 \mu \mathrm{OD}^{30}$ This noise level can be seen directly by forming the difference spectrum $d$-e (middle panel), thus canceling most of the population background. Successiveshot referencing (again assuming $\gamma_{\text {consec }} \approx 0.92$ ) would not only need $10 \times$ longer, but also not all differences $i-j$ can be formed. Working on other molecular systems, Mathies et $a l .{ }^{28}$ derived a spectral reference from same shots but then time-averaged the result prior to forming pertinent differences or quotients; they needed $18 \mathrm{~s}$ acquisition time to reach equivalent noise.

In summary: large fluctuations of the probe light can be canceled by spectral referencing on a shot-by-shot basis. For this purpose, an optical setup was presented which enables 
equivalent energy transmission through the sample and reference paths. An improvement of $\mathrm{S} / \mathrm{N}$ is generally achieved compared to measurements where subsequent pulses are used for referencing.

\section{ACKNOWLEDGMENTS}

We are grateful to the Deutsche Forschungsgemeinschaft for support (SFB 450 and Cluster of Excellence "Unifying Concepts in Catalysis").

${ }^{1}$ A. N. Tarnovsky, W. Gawelda, M. Johnson, C. Bressler, and M. Chergui, J. Phys. Chem. B 110, 26497 (2006).

${ }^{2}$ A. V. Zamyatin, A. V. Soldatova, and M. A. J. Rodgers, Inorg. Chim. Acta 360, 857 (2007).

${ }^{3}$ R. Huber, T. Köhler, M. O. Lenz, E. Bamberg, R. Kalmbach, M. Engelhard, and J. Wachtveitl, Biochemistry 44, 1800 (2005).

${ }^{4}$ T. A. Zeidan, Q. Wang, T. Fiebig, and F. D. Lewis, J. Am. Chem. Soc. 129, 9848 (2007).

${ }^{5}$ J. L. Pérez Lustres, A. L. Dobryakov, A. Holzwarth, and M. Veiga, Angew. Chem., Int. Ed. 46, 3758 (2007).

${ }^{6}$ C. Ley, J. Brazard, F. Lacombat, P. Plaza, M. M. Martin, G. A. Kraus, and J. W. Petrich, Chem. Phys. Lett. 457, 82 (2008).

${ }^{7}$ P. Z. El-Khoury and A. N. Tarnovsky, Chem. Phys. Lett. 453, 160 (2008).

${ }^{8}$ S. A. Kovalenko, A. L. Dobryakov, I. Ioffe, and N. P. Ernsting, Chem. Phys. Lett. 493, 255 (2010).

${ }^{9}$ R. R. Alfano, The Supercontinuum Laser Source (Springer, Berlin, 2005).

${ }^{10}$ D. Polli, L. Lüer, and G. Cerullo, Rev. Sci. Instrum. 78, 103108 (2007).

${ }^{11}$ U. Megerle, I. Pugliesi, C. Schriever, C. F. Sailer, and E. Riedle, Appl Phys. B: Lasers Opt. 96, 215 (2009).

${ }^{12}$ J. A. Moon, Rev. Sci. Instrum. 64, 1775 (1993).

${ }^{13}$ C. Schriever, S. Lochbrunner, E. Riedle, and D. J. Nesbitt, Rev. Sci.
Instrum. 79, 013107 (2008)

${ }^{14}$ E. C. Carroll, M. P. Hill, D. Madsen, K. R. Malley, and D. S. Larsen, Rev. Sci. Instrum. 80, 026102 (2009).

${ }^{15}$ P. J. M. Johnson, V. I. Prokhorenko, and R. J. D. Miller, Opt. Express 17, 21488 (2009).

${ }^{16}$ A. Kutter, The Schiefspiegler (Oblique Telescope). Bulletin A of Sky and Telescope (Sky Publishing, Cambridge, 1958).

${ }^{17}$ T. Bultmann and N. P. Ernsting, J. Phys. Chem. 100, 19417 (1996).

${ }^{18}$ With strongly absorbing samples, a stationary copy of the sample cell should be placed just before entrance into the reference spectrograph.

${ }^{19}$ The sample must not degrade photochemically at the windows. With this provision, oscillation of the cell had a negligible influence on $\mathrm{S} / \mathrm{N}$.

${ }^{20}$ P. R. Bevington and D. K. Robinson, Data Reduction and Error Analysis for the Physical Sciences, 2nd ed. (McGraw-Hill, New York, 1992).

${ }^{21}$ The cited correlation of 0.916 refers to the integrated probe pulse energy, whereas our value 0.992 refers to the probe energy in an individual wavelength increment.

${ }^{22}$ M. Sajadi, K. E. Furse, X. Zhang, S. A. Kovalenko, S. A. Corcelli, and N. P. Ernsting (in preparation).

${ }^{23}$ N. P. Ernsting, S. A. Kovalenko, T. Senyushkina, J. Saam, and V. Farztdinov, J. Phys. Chem. A 105, 3443 (2001).

${ }^{24}$ S. A. Kovalenko, A. L. Dobryakov, J. Ruthmann, and N. P. Ernsting, Phys. Rev. A 59, 2369 (1999).

${ }^{25}$ R. Penrose, Proc. Cambridge Philos. Soc. 52, 17 (1956).

${ }^{26}$ With a large number of exponential terms, all systematic change can of course be described. Therefore the existence of an oscillation is equivalent to the statement that an oscillatory residual remains from a global fit, over the limited range of 0-9 ps, with four to five exponentials.

${ }^{27}$ P. Kukura, S. Yoon, and R. A. Mathies, Anal. Chem. 78, 5952 (2006).

${ }^{28}$ D. W. McCamant, P. Kukura, S. Yoon, and R. A. Mathies, Rev. Sci. Instrum. 75, 4971 (2004).

${ }^{29}$ A. Weigel and N. P. Ernsting, J. Phys. Chem. B 114, 7879 (2010).

${ }^{30}$ Equivalent to recording a transient absorption spectrum with $25 \mu \mathrm{OD} \mathrm{rms}$ in $1 \mathrm{~s}$. 